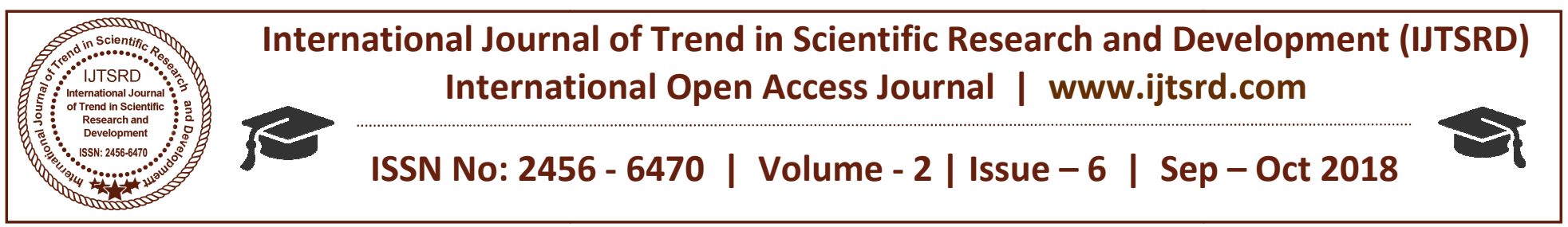

\title{
An Attendance Monitoring System Using Biometric Technology and GSM Module
}

\author{
Bilal Hussain Ch, Subayyal \\ Research Scholars, Department of Computer Science and Engineering, \\ University of Engineering \& Technology, Lahore, Pakistan
}

\section{ABSTRACT}

Biometric technology that involves the identification and verification of the individuals by analyzing finger print is now being used in the various and wide aspects of our life. Biometric system includes various identification and verification methods in which the finger print detection is one of the most widely used and accepted method. In this paper we are proposing and developing a system which includes finger print detection and GSM module for the attendance and for the report generation of the students.

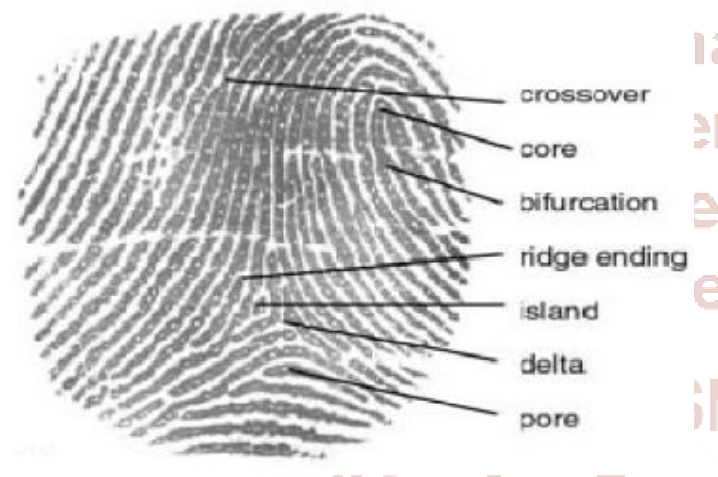

We have developed various algorithms that convert the image of the finger in to the binary form and store it in the database. We are extracting features of the finger print and we are identifying finger print on the basis of these extracted features. We have developed a whole system in which first the student is registered and then authenticated and the attendance is counted and at the end the report is generated and that report will be send to the parents of the students.

Keyword: Finger print, GSM module, Biometric technology.

\section{INTRODUCTION}

Attendance management system is the easiest way to keep track of the employers and the students for an organization or an institution. AMS system is now widely used and the most reliable process for keeping the track of the students and the employers.

Old conventional methods are still being used in most of the industries and institutions. This conventional method has major flaws in it and is not reliable. The major flaw is that the attendance of an absent student is also marked by his/her friends. And this type of system is still very difficult to manage and handle. It's also very troublesome for the lecturer to make count the attendance manually and to generate report at the end. The report generated at the end is obviously not accurate because of the human error. Technology has emerged very fast and various techniques has been created to help us out form such problems. We have created such a method in which the attendance of the student would automatically be counted at the end and report will also be created and will be send to the parents of the student.

We are basically using a finger print detector and the GSM module for performing the functionality in our system. We are using the biometric technology in which the finger print of the student identified and verified and the other working is carried out using this basic method. Both the modules (GSM and the finger print recognizer) have the different and viral importance in the main stream of the process.

The basic steps in our process are:

\section{Identification:-}

This is the first process in which finger print of the student is identified. At the time of the enrollment the finger print of the student is required and the students have to place the finger on the finger print device so that the device gets a fair good image of the finger. 
The student is asked to place the finger on the finger print device three times so that the correct image of the finger is placed in to the database.

\section{Verification:-}

This is the second process in which the finger print of the student is verified and checked with the image placed in the database if the image in the database matches the one getting from the device the student is verified and the attendance for that day is counted. And the student has to give the print of the finger each day to make himself / herself count for that day.

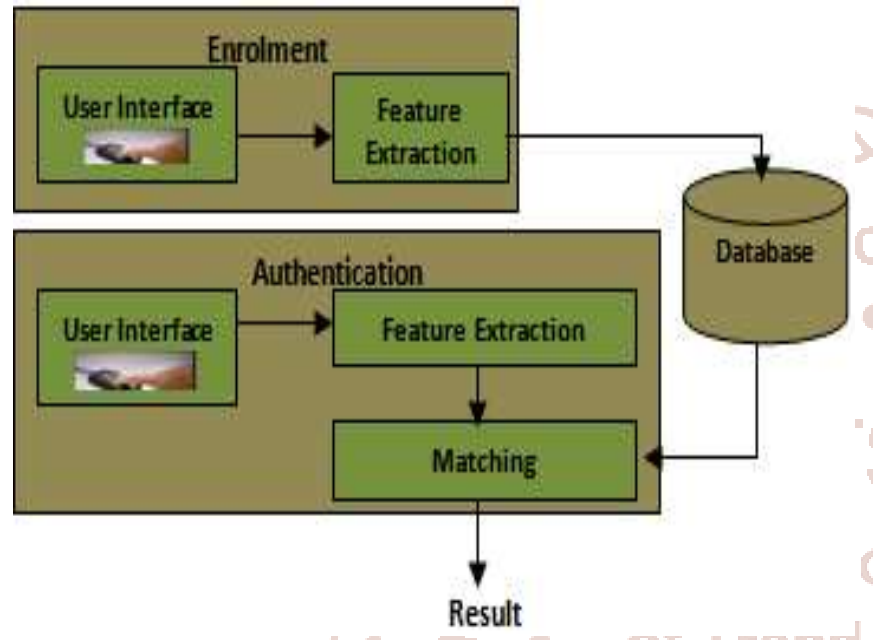

\section{Report generation:-}

At the end of the day, the report is created for that day and at the end of the month the report is created for that month. The proceeding for each day is saved and the report id generated at the end of the month automatically at the generated report is send to the parents of the students.

We have developed entire procedure using C\# language.

\section{Hardware used:-}

The hardware used in our system is:-

\section{GSM module:-}

The GSM module is used for the sending the report generated to the parents of the specified students.

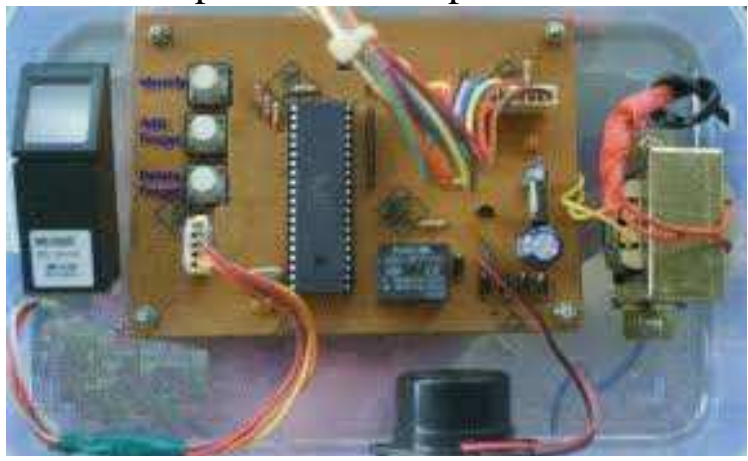

We have coded the hardware of GSM using the language $\mathrm{C \#}$.

\section{Finger print detector:-}

We used the finger print scanner/detector for the identification and the verification of the finger print of the student.

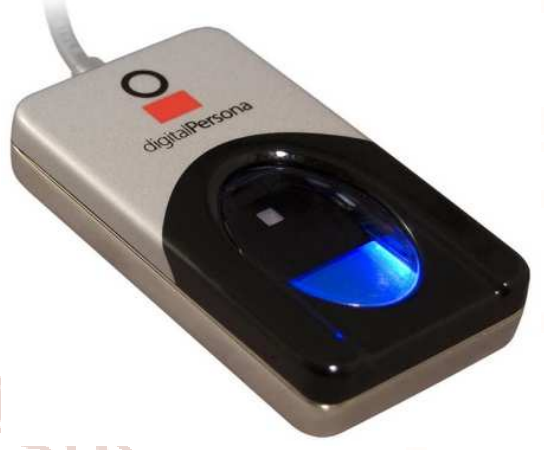

\section{System architecture:-}

Our system consists of two basic modules and three different phases. Two of the basic modules are finger print scanner and the GSM module. And three phases are identification, verification and report generation. SY T E M A R C H IT E C T U R E

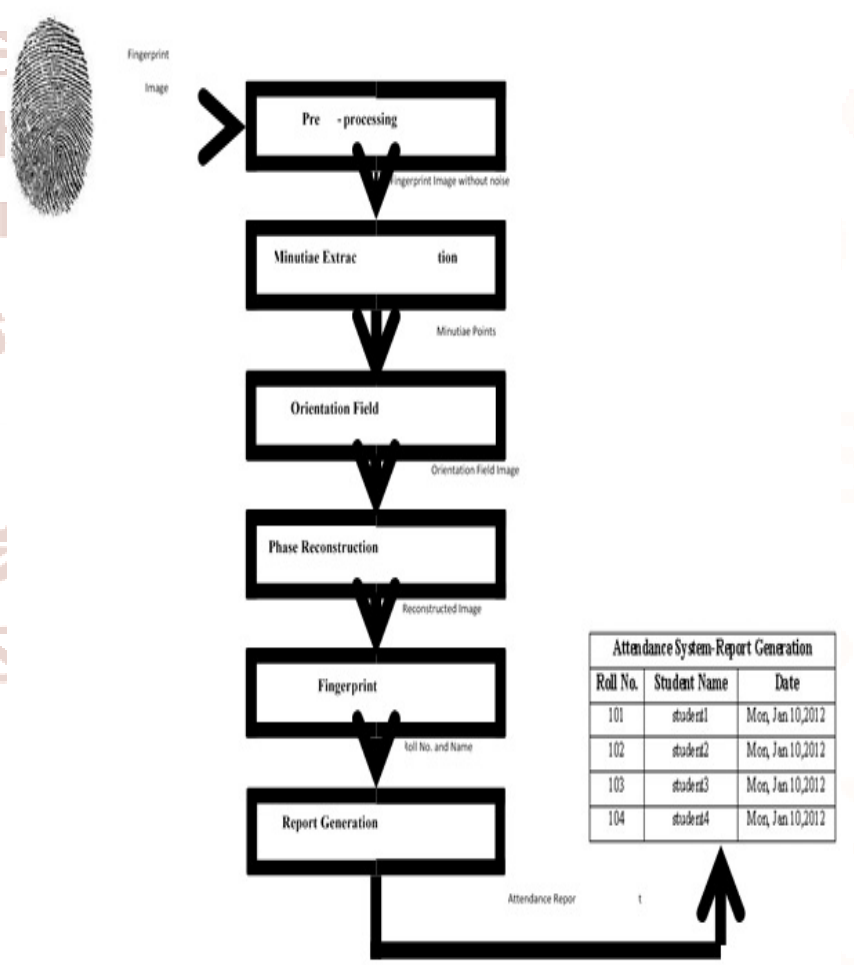

As it is elaborated in the pictorial representation of the system architecture that we have different phases and algorithms to make our system work efficient.

First there is the finger print acceptance from the device and the print is stored in the database. Then there is the extraction of the minutiae features from 
the print that is received from the device. After that there is orientation field and after that we have the phase reconstruction of the print by all these steps the print obtained will be sharp and accurate then this image is stored in the database. After that report is generated and the report is send to the parents using GSM module.

\section{Explained view of the system:-}

In the system we have basically three different phases.

\section{Identification phase:-}

In this phase the image is obtained from the finger print recognition device and stored in the database. The internal working of this step needs a little elaboration.

Steps involved in this are

$>$ Pre-processing
$>$ Minutiae extraction.
$>$ Orientation field
$>$ Phase reconstruction
$>$ Image formed
$>$ Saved in the database.

As the image is received pre-processing for the image take place .Which includes the improvement of the image by removing the dust particles and the other effects like water effect or dust effect. After that minutiae extraction for the image take place. Features are extracted to identify the image, clear and visible features are selected on the bases of which the print identified.

After that there is orientation field and phase reconstruction and the accurate image is created and placed in the database.

The image is stored in the database in binary form. We have developed different MATLAB algorithms to carry out the process in an efficient manner that I have explained.

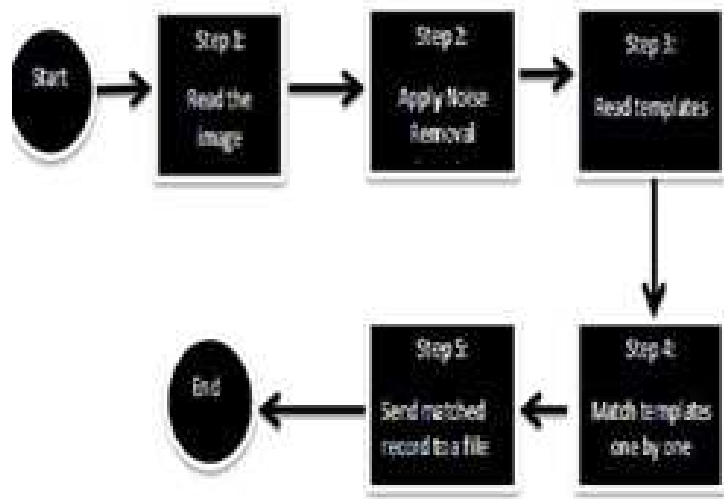

Block diagram of our first process.
Verification phase:-

In this phase the attendance of the student is marked. This process includes Finger print scanner. In this phase the student is asked to place the finger on the finger print device in order to mark its attendance.

The student will place the finger and the finger print of the student is checked with all the finger prints in the database. If the print is matched the attendance for the day of the specified student is marked. And if in case the print doesn't match the system will generate the error that the student is not registered and authorized.

This process carries on for the whole of the month. And for the each day the attendance is marked and updated in database.

The database is maintained and updated regularly.

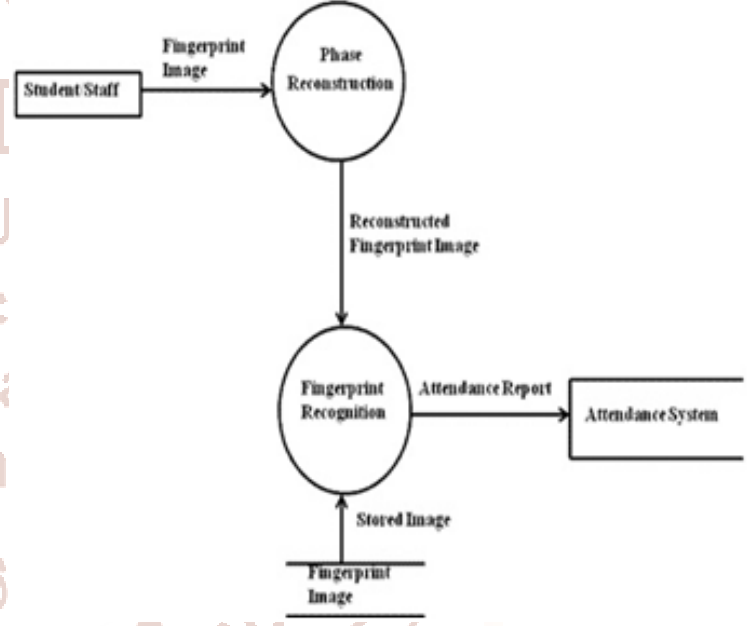

Block diagram of the two phases

\section{Report generation:-}

In this phase the report that is maintained and updated regularly and is placed in the database will be send to the parents of the students. This phase includes the use of the GSM module.

The report will be send to the number that is registered at the time of enrollment of the student. The report will be generated automatically. The language used is $\mathrm{C \#}$.

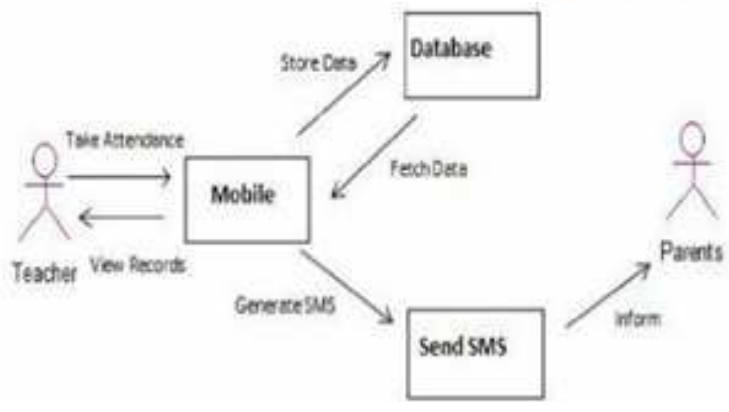


Benefits of our methodology:

The benefits of our methodology are:

\section{$>$ Fairness:}

Our system will introduce fairness in the attendance process to next level. As the methodology proposed by us is nearly impossible to break. So it will introduce fairness in the attendance process.

\section{$>$ Increases productivity:-}

Our system will increase the productivity of the staff as they don't have to manually mark the attendance. It will save the time and effort that can be used in other aspects.

\section{$>$ Uniqueness:-}

We have defined uniqueness in our system. We have used MATLAB functions that help the administrator to sharp the image and help to get a clear view of the print. We also have developed MATLAB functions for this process.

$>$ Satisfaction of the parents :-

The main essence of our system is that parents will get the monthly report about their child. They will be fully aware of the on goings of their child. The report will automatically send so there would be no error what so ever.

$>$ Accuracy:-

Our methodology is accurate. We have introduced accurate and dependable devices in the system. They will provide the most accurate result is all conditions.

\section{$>$ Efficiency:-}

Efficiency is the important factor that we have considered.

Our system will enhance the efficiency to the next level. Our methodology will provide the result in minimal time and with precision. matching techniques. As I have explained earlier that we have developed efficient algorithms in the MATLAB for the working of our system that there is not even a minute chance that there will be error.

Besides that we have paid most of the attention on the accuracy. We have coded the accurate and efficient code that it will withstand all the conditions. After that we have used advanced security methods so that the unauthorized person cannot get into our system.

We have used and developed greedy algorithms that outputs the most accurate and most precise result.

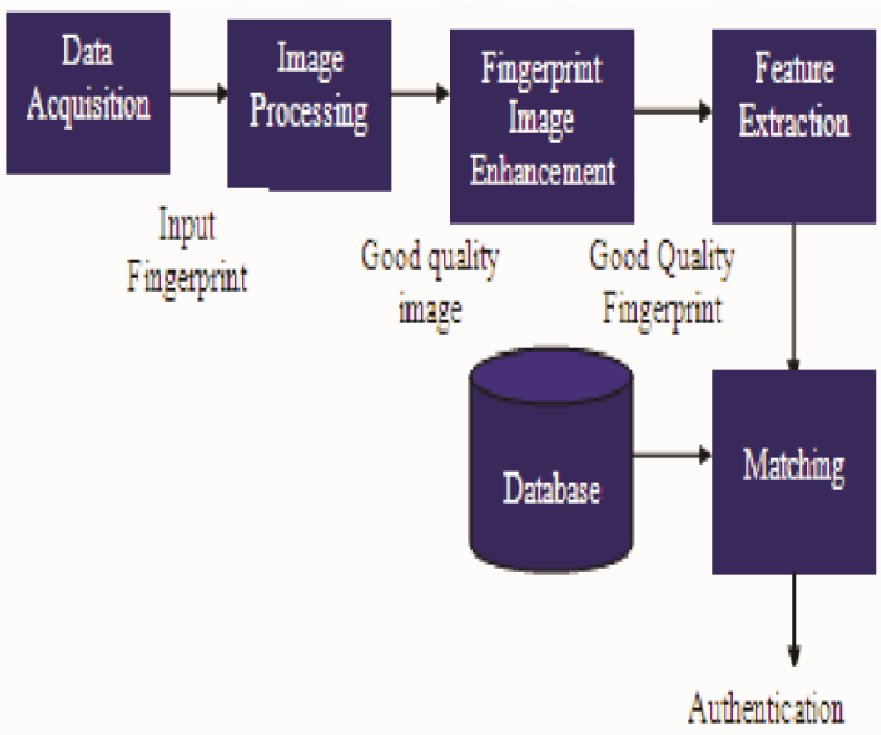

\section{Conclusion:-}

We have developed a system in which includes three basic phases. Registration phase or the identification phase and the second phase is authentication phase and the third process is report generation.

\section{Future work:-}

In the future the system can be enhanced by using other biometric techniques.

The main difference in our system and the others systems in the market is efficiency. We have used advanced techniques for the features extraction and

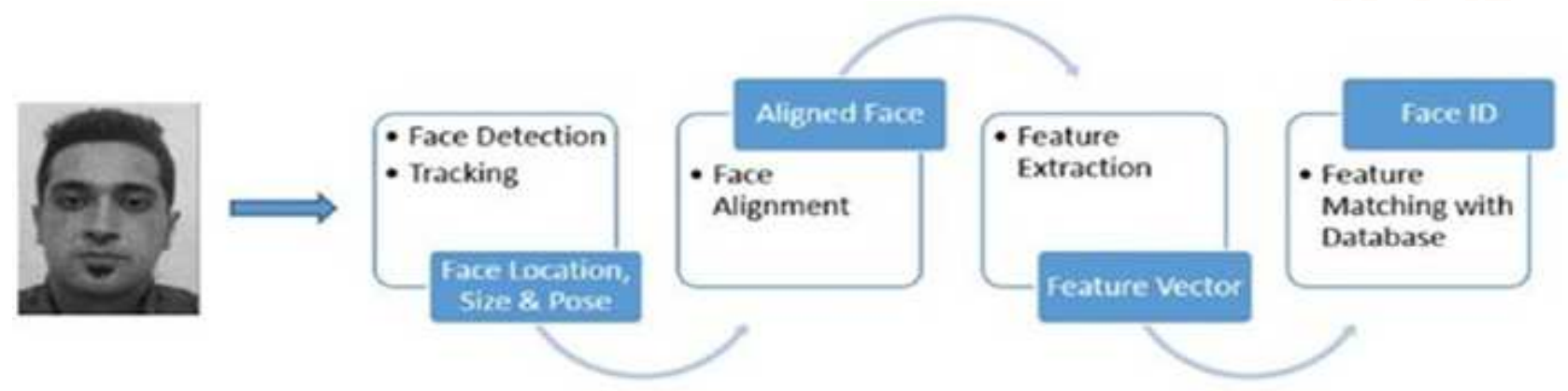


Face recognition can also be used to develop such a system. The facial expression and the features can be extracted and can be placed in the database on the basis of which the system matches the facial expression of the students with the one saved in the database and will generate the report.

Besides that iris detection can also be used for the attendance system. The iris features can be extracted and will be placed in the database and the system matches the features with the one places in the database. And the report will be generated at the end.

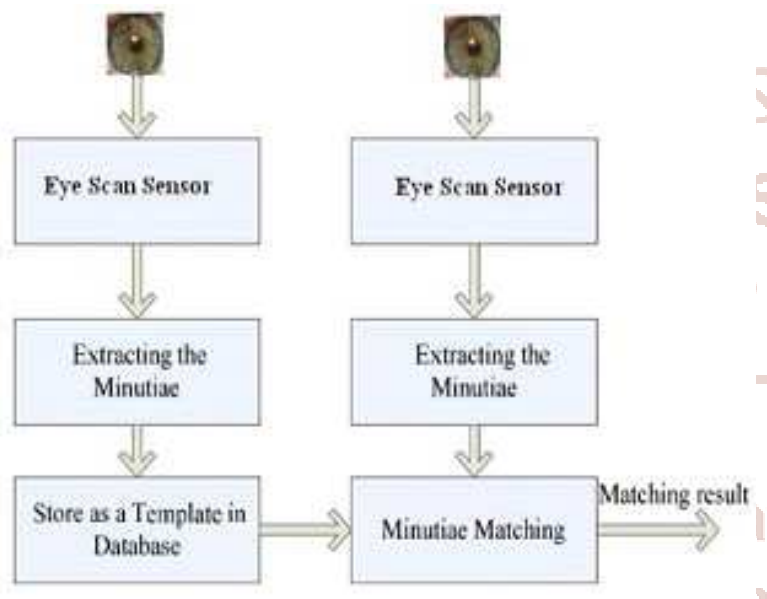

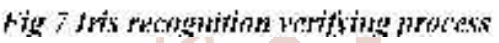

\section{References:-}

1. Kadry, Seifedine, and Mohamad Smaili. "Wireless attendance management system based on iris recognition." Scientific Research and essays 5.12 (2010): 1428-1435.

2. Rao, G. Sambasiva, etal. "A Novel Fingerprints Identification System Based on the Edge Detection." International Journal of Computer Science and Network Security 8 (2008): 394-397.

3. Shoewu, O., and O. A. Idowu. "Development of attendance management system using biometrics." The Pacific Journal of Science and Technology 13.1 (2012).

4. Taxila, Punjab. "Development of Academic Attendence Monitoring System Using Fingerprint Identification." IJCSNS 9.5 (2009): 164.

5. Jain, Anil K., Arun Ross, and Salil Prabhakar. "An introduction to biometric recognition." Circuits and Systems for Video Technology, IEEE Transactions on 14.1 (2004): 4-20.

6. Paik, Michael, et al. "A biometric attendance terminal and its application to health programs in India." Proceedings of the 4th ACM Workshop on Networked Systems for Developing Regions. ACM, 2010. 